\title{
The effect of tissue-engineered cartilage biomechanical and biochemical properties on its post-implantation mechanical behavior
}

\author{
Mehdi Khoshgoftar • Wouter Wilson • Keita Ito • \\ Corrinus C. van Donkelaar
}

Received: 29 August 2011 / Accepted: 14 February 2012 / Published online: 3 March 2012

(C) The Author(s) 2012. This article is published with open access at Springerlink.com

\begin{abstract}
The insufficient load-bearing capacity of today's tissue-engineered (TE) cartilage limits its clinical application. Focus has been on engineering cartilage with enhanced mechanical stiffness by reproducing native biochemical compositions. More recently, depth dependency of the biochemical content and the collagen network architecture has gained interest. However, it is unknown whether the mechanical performance of TE cartilage would benefit more from higher content of biochemical compositions or from achieving an appropriate collagen organization. Furthermore, the relative synthesis rate of collagen and proteoglycans during the TE process may affect implant performance. Such insights would assist tissue engineers to focus on those aspects that are most important. The aim of the present study is therefore to elucidate the relative importance of implant ground substance stiffness, collagen content, and collagen architecture of the implant, as well as the synthesis rate of the biochemical constituents for the post-implantation mechanical behavior of the implant. We approach this by computing the post-implantation mechanical conditions using a composition-based fibril-reinforced poro-viscoelastic swelling model of the medial tibia plateau. Results show that adverse implant composition and ultrastructure may lead to post-implantation excessive mechanical loads, with collagen orientation being
\end{abstract}

\footnotetext{
M. Khoshgoftar · W. Wilson · K. Ito · C. C. van Donkelaar $(\varangle)$ Department of Biomedical Engineering, Eindhoven University of Technology, PO Box 513, $5600 \mathrm{MB}$, Eindhoven, The Netherlands e-mail: c.c.v.donkelaar@tue.nl

M. Khoshgoftar

e-mail: m.khoshgoftar@tue.nl

W. Wilson

e-mail:w.wilson@tue.nl

K. Ito

e-mail: k.ito@tue.nl
}

the most critical variable. In addition, we predict that a faster synthesis rate of proteoglycans compared to that of collagen during TE culture may result in excessive loads on collagen fibers post-implantation. This indicates that even with similar final contents, constructs may behave differently depending on their development. Considering these aspects may help to engineer TE cartilage implants with improved survival rates.

Keywords Tissue engineering · Implantation · Collagen architecture $\cdot$ Finite element modeling

\section{Introduction}

Among the main limitations of therapies such as chondroplasty for treatment for local cartilage damage is the lack of donor tissue as well as the lack of grafts that contain biologically and mechanically functional tissue (Schek et al. 2004). Tissue engineering (TE) approaches have the potential to overcome these limitations (Risbud and Sittinger 2002; Kuo et al. 2006; Nesic et al. 2006; Noth et al. 2008). Despite promising improvements, the insufficient load-bearing capacity of today's TE cartilage is an important limiting factor in its clinical application. Physiologic mechanical forces are thought to induce long-term implant failure (Scotti et al. 2007). The success rate of cartilage replacement by TE implants is thought to be enhanced if the implant had the functional load-bearing properties of the native tissue.

Native cartilage derives its compressive properties from the interaction between proteoglycans (PG's), which attract water through osmotic pressure, and collagen fibers, which resist tension. The functional significance of the specific physiological depth-dependent collagen structure in native tissue (with vertical fibers in the deep zone and horizontal fibers in the superficial zone) is well emphasized in the 
literature (Korhonen et al. 2002; Owen and Wayne 2006; Wilson et al. 2007; Korhonen et al. 2008; Shirazi and ShiraziAdl 2008; Shirazi et al. 2008). Vertical fibrils in the deep zone resist swelling (Wilson et al. 2006b) and protects the solid matrix against large strains at the subchondral junction (Shirazi and Shirazi-Adl 2008). The superficial zone plays a crucial role in resisting elevated tensile stresses parallel to the articular surface (Owen and Wayne 2006). Experimental and numerical studies have demonstrated that the removal of the superficial zone from normal cartilage reduces the ability of the tissue to support axial loads and retain fluids (Glaser and Putz 2002; Torzilli et al. 1983; Torzilli 1993; Owen and Wayne 2006). Given these important roles of ultrastructure in load-bearing capabilities of native tissue, one of the main challenges for tissue engineering of mechanically stable cartilage is to find the cues to create an engineered tissue with the ultrastructure components and organizations similar to those of native tissue.

Using mechanical stimuli to enhance matrix production in TE constructs, it is possible to tissue engineer cartilage with almost native PG's content. However, collagen reaches only $15-35 \%$ of the native content after 5-12 weeks (Eyrich et al. 2007; Hu and Athanasiou 2006; Miot et al. 2006), and the native collagen network architecture is not reproduced. In particular, Kelly et al. (2006) observed that under unconfined compression, the most common loading regime in cartilage TE, collagen fibers align perpendicular to the loading direction rather than in physiological architecture. Approaches to create a depth-dependent tissue include using depth-dependent scaffold properties or cell sources. Unfortunately, these approaches had limited success in the (mid)long-term (Kim et al. 2003; Malda et al. 2005; Ng et al. 2005, 2009; Klein et al. 2007; Moutos et al. 2007). Recently, depth dependency of the collagen network architecture has gained interest in cartilage TE (Kock et al. 2010; Khoshgoftar et al. 2011). However, it is unknown whether the mechanical performance of TE cartilage after implantation would benefit more from higher biochemical compositions content of the implant or from achieving an appropriate depthdependent collagen organization. This is important to understand because engineering a tissue with more matrix content would require a different experimental approach than engineering a tissue with depth-dependent structure or with physiological collagen architecture (Khoshgoftar et al. 2011).

Regarding biochemical constituents, not only their content but also the rate of their synthesis during TE culture may influence mechanical behavior of engineered tissues. Indeed, previous experimental studies showed significant influences of the synthesis rate of PGs and collagen on mechanical properties of the cartilage explants (Asanbaeva et al. 2007) and TE constructs (Bian et al. 2009). Effects of such phenomena on post-implantation mechanical performance of TE implants have not been reported so far, and it is unknown how different synthesis rate of the PGs and collagen during TE process may change mechanical conditions in TE implants after implantation.

Insight into the relative importance of the above-mentioned aspects would assist tissue engineers to focus on those aspects that are likely to improve implant performance most. The aim of the present study is, therefore, to elucidate the relative importance of ground substance stiffness, collagen content, and collagen architecture of the implant for the postimplantation mechanical performance of the implant. Furthermore, we evaluate how implant performance depends on the relationship between PG and collagen synthesis rates during TE. Implants with various composition and structural organizations, implanted in intact cartilage with native contents and a physiological collagen architecture, are examined to elucidate which particular aspect of the implant is to be improved for a better match between implant and host tissue.

\section{Methods}

\subsection{General approach}

We investigated the role of ground substance stiffness, collagen content, and collagen architecture of TE implants on the post-implantation mechanical condition of the implant when the implant was attached to the native tissue and mechanically loaded. Mechanical condition of the implant was compared against the mechanical condition of loaded native tissue without implant. A numerical approach was used, because it is not feasible/possible to experimentally examine implants with different ultrastructure and collagen organization and evaluate mechanical strains and stresses throughout the implants.

To evaluate the importance of the collagen network architecture, the direction of the collagen fibers was varied in the implant, such that they were random, horizontal, and physiological. To evaluate the importance of the collagen content and ground substance stiffness, for each implant type, implants with three different constituents were compared; first, implant with $1 / 2$ native non-fibrilar ground substance stiffness and 1/4 native collagen content, which represents the currently producible collagen content in TE studies; second, implant with native ground substance stiffness and $1 / 4$ native collagen content; third, implant with $1 / 2$ native ground substance stiffness and 3/4 native collagen content. List of the evaluated cases is summarized in Table 1.

\subsection{Finite element mesh and material model}

We created an axisymmetric finite element model (ABAQUS v6.9, Pawtucket, RI, USA) of the medial tibia plateau cartilage in which a cylindrical part of the mesh at the central region of the model represented a TE implant (Fig. 1). A validated composition-based fibril-reinforced poro-viscoelastic 
Table 1 List of the evaluated ratio of the implants GAG content, matrix stiffness, and collagen content to those in native tissue for implants with random, horizontal, and native-like primary fibers

\begin{tabular}{lll}
\hline $\begin{array}{l}\text { Implant collagen } \\
\text { architecture }\end{array}$ & $\begin{array}{l}\text { Implant ground substance } \\
\text { stiffness/native ground } \\
\text { substance stiffness }\end{array}$ & $\begin{array}{l}\text { Implant collagen } \\
\text { content/native } \\
\text { collagen content }\end{array}$ \\
\hline Random & $1 / 2$ & $1 / 4$ \\
& 1 & $1 / 4$ \\
Horizontal & $1 / 2$ & $3 / 4$ \\
& $1 / 2$ & $1 / 4$ \\
& 1 & $1 / 4$ \\
Physiological & $1 / 2$ & $3 / 4$ \\
& $1 / 2$ & $1 / 4$ \\
& $1 / 2$ & $1 / 4$ \\
\hline
\end{tabular}

swelling material model was adopted in which the porous matrix of the biphasic tissue consisted of a swelling nonfibrillar ground substance, which contains mainly PG's and TE scaffold substance, and a fibrillar part representing the collagen network (Wilson et al. 2004, 2006b, 2007). The mechanical behavior of the material model was the direct consequence of the composition (fluid fraction, collagen fraction, fixed charge density) and the structure (collagen orientation) of the tissue. The governing stress equation was (Wilson et al. 2007):

$$
\begin{aligned}
\boldsymbol{\sigma}_{t o t}= & -\mu^{f} \mathbf{I}+n_{s, 0}\left(\left(1-\sum_{i=1}^{\text {totf }} \rho_{c}^{i}\right) \boldsymbol{\sigma}_{n f}+\sum_{i=1}^{\text {totf }} \rho_{c}^{i} \boldsymbol{\sigma}_{f}^{i}\right) \\
& -\Delta \pi \mathbf{I}
\end{aligned}
$$
$\Delta \pi$ the osmotic pressure gradient, $n_{s, 0}$ the initial solid volume (in the unloaded and non-swollen state), $\sigma_{n f}$ the stress in the non-fibrillar ground substance, $\sigma_{f}^{i}$ the fibril stress in

\section{(a)}

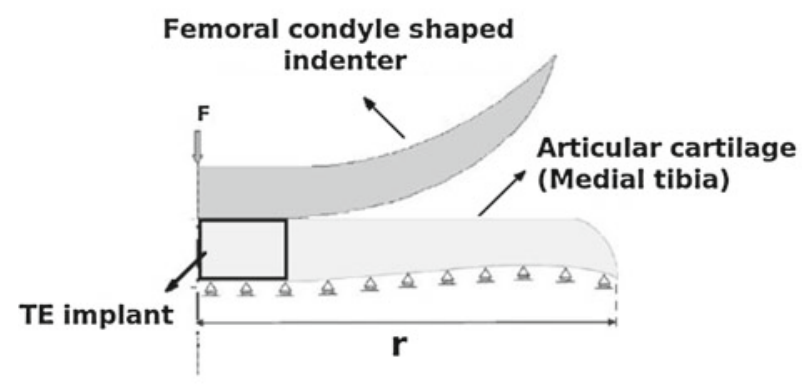

where $\mu^{f}$ was the water chemical potential, $\boldsymbol{I}$ the unit tensor,

the $i$ th fibril direction, $\rho_{c}^{i}$ the volume fraction of the nonlinear, viscoelastic collagen fibrils with respect to the total solid volume, $i$ detonated the number of the fibril compartment, and totf the total number of the fibrils. In all cases, PG's content in the implant was assumed to be $75 \%$ of the native tissue in agreement with experimental studies (Kelly et al. 2006). Expressions for osmotic swelling pressure can be found in Wilson et al. (2006b). Permeability was chosen to be twice that in native tissue (Buschmann et al. 1992; Owen and Wayne 2006). The composition and mechanical properties used for native tissue were the same as in Wilson et al. (2006b). The material model was implemented in ABAQUS using the user subroutine UMAT.

\subsubsection{Non-fibrillar ground substance}

For the non-fibrillar ground substance, a compressible NeoHookean model was used of which the compressibility was dependent on the solid fraction by the following expression for the Poisson's ratio (Wilson et al. 2007):

$v_{m}=0.5 n_{s}=0.5 \frac{n_{s, 0}}{J}$

where $J$ was the determinant of the deformation gradient tensor $\boldsymbol{F}$. Having the following expressions for the bulk modulus $K_{m}$ and the shear modulus $G_{m}$ as a function of the Young's Modulus $E_{m}$, and the Poisson's ratio $v_{m}$ as:

$\begin{aligned} K_{m} & =\frac{E_{m}}{3\left(1-2 v_{m}\right)} \\ G_{m} & =\frac{E_{m}}{2\left(1+v_{m}\right)}\end{aligned}$

$K_{m}$ can be expressed as:

$K_{m}=\frac{2}{3} G_{m} \frac{\left(1+0.5 n_{s, 0} / J\right)}{\left(1-n_{s, 0} / J\right)}$

The Cauchy stress of the non-fibrillar ground substance was given by (Wilson et al. 2007):

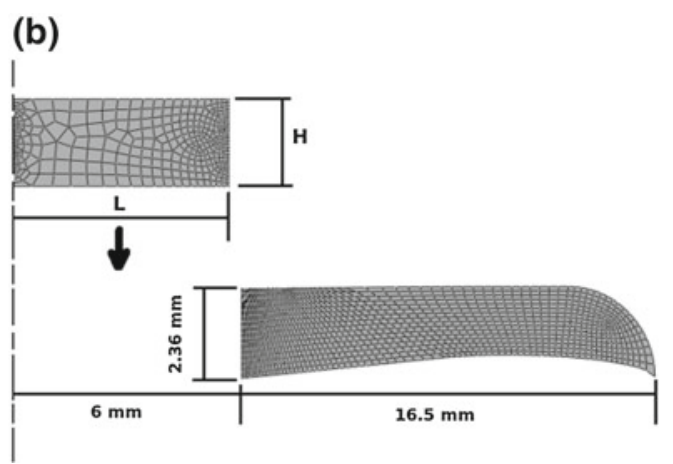

Fig. 1 a Axisymmetric finite element model of the medial tibia plateau cartilage with a TE implant located at the central region, b Finite element mesh of the implant (top) with initial height $\mathrm{H}$ and length $\mathrm{L}$ before swelling equilibrium and native host cartilage (bottom) with a central hole with radius of $6 \mathrm{~mm}$ and height of $2.36 \mathrm{~mm}$ 


$$
\begin{aligned}
\sigma_{n f}= & -\frac{1}{6} \frac{\ln (J)}{J} G_{m} \mathbf{I}\left[-1+\frac{3\left(J+n_{s, 0}\right)}{\left(-J+n_{s, 0}\right)}+\frac{3 \ln (J) J n_{s, 0}}{\left(-J+n_{s, 0}\right)^{2}}\right] \\
& +\frac{G_{m}}{J}\left(\mathbf{F} \cdot \mathbf{F}^{T}-J^{2 / 3} \mathbf{I}\right)
\end{aligned}
$$

\subsubsection{Collagen fiber network; material model and architecture}

To capture the nonlinear strain-dependent viscoelastic behavior of collagen fibers, the mechanical behavior of the fibers was represented by two parallel nonlinear springs, $P_{1}$ and $P_{2}$, and a dashpot with damping coefficient $\eta$ in series with $P_{2}$. For the springs, two-parameter exponential stress-strain relationship was used (Wilson et al. 2006b):

$$
\begin{array}{ll}
P_{1}=E_{1}\left(e^{k_{1} \varepsilon_{f}}-1\right) & \text { for } \varepsilon_{f}>0 \\
P_{1}=0 & \text { for } \varepsilon_{f} \leq 0 \\
P_{2}=E_{2}\left(e^{k_{2} \varepsilon_{e}}-1\right) & \text { for } \varepsilon_{e}>0 \\
P_{2}=0 & \text { for } \varepsilon_{e} \leq 0
\end{array}
$$

with $E_{1}, E_{2}, k_{1}$ and $k_{2}$ positive material constants and $\varepsilon_{f}$, the total fibril logarithmic strain and $\varepsilon_{e}$, the logarithmic strain in the spring $P_{2}$.

Since the strains in the upper and lower part of the spring system were the same, the total fibril stress was given by:

$$
\begin{array}{lll}
P_{f}=P_{1}+P_{2} & \text { for } & \varepsilon_{f}>0 \\
P_{f}=0 & \text { for } & \varepsilon_{f} \leq 0
\end{array}
$$

The time derivative of the fibril strain was given by:

$\dot{\varepsilon}_{f}=\frac{\dot{P}_{2}}{\left(P_{2}+E_{2}\right) k_{2}}+\frac{P_{2}}{\eta}$

Determination of the viscoelastic fibril stress can be found in Wilson et al. (2006b).

Due to deformation, the surface associated with a fibril changes. To include this, the fibril stress was expressed with reference to its original state. The 2nd Piola-Kirchhoff stress tensor was given by Wilson et al. (2006b, 2007):

$\mathbf{S}_{f}=S_{f} \vec{e}_{f, 0} \vec{e}_{f, 0}$

where $\vec{e}_{f, 0}$ was the initial fibril direction. The 2nd PiolaKirchhoff stress in the initial fibril direction $S_{f}$ was given by:

$S_{f}=\frac{P_{f}}{\lambda}=\frac{P_{f}}{\left\|\mathbf{F} \cdot \vec{e}_{f, 0}\right\|}$,

where $\lambda$ was the elongation of the fibril. The total Cauchy stress expressed as a function of the deformed state was given by:

$\sigma_{f}=\frac{1}{J} \mathbf{F} \cdot \mathbf{S}_{f} \cdot \mathbf{F}^{T}=\frac{1}{J} \frac{P_{f}}{\lambda} \mathbf{F} \cdot \vec{e}_{f, 0} \vec{e}_{f, 0} \cdot \mathbf{F}^{T}=\frac{\lambda}{J} P_{f} \vec{e}_{f} \vec{e}_{f}$ where $\overrightarrow{e_{f}}$ was the current fibril direction and the factor $\lambda / J$ represents the change in surface associated with the fibril.

For more details on the fibers material model, the reader is referred to Wilson et al. (2004, 2006b, 2007). Based on Wilson et al. (2006b, 2007), the material constants we used were $E_{1}=4.316 \mathrm{MPa}, E_{2}=19.97 \mathrm{MPa}, k_{1}=16.85, k_{2}=$ 41.49 , and $\eta=1.424 \times 10^{5} \mathrm{MPa}$ s.

A collagen network with physiological architecture was captured by prescribing the local orientation of primary collagen fibrils in each integration point, such that they extended perpendicular from the subchondral bone in the deep zone, curve over in the transitional zone, and flush with the articular surface in the superficial zone. In addition, seven secondary fibrils were included per integration point: three running in the directions of the $r-, y$-, and $z$-axis in the axisymmetric configuration, and four running in directions between those axes. The relative collagen fractions were then given by:

$$
\begin{array}{ll}
\rho_{c}=\rho_{c, \text { tot }} \frac{C}{\text { totf }}=\rho_{c, \text { tot }} \frac{C}{2 C+7} & \text { for the primary fibrils } \\
\rho_{c}=\rho_{c, \text { tot }} \frac{1}{\text { totf }}=\rho_{c, \text { tot }} \frac{1}{2 C+7} & \text { for the secondary fibrils }
\end{array}
$$

where $C$ was a positive constant, $\rho_{c, t o t}$ the depth-dependent total collagen volume fraction per total solid volume.

In the case of constructs with collagen fibers predominantly oriented parallel to the articular surface, primary fibers were considered to be horizontal throughout the entire depth of the construct, and $C$ was chosen 3.0 to represent the relative importance of primary fibrils over secondary fibrils. In the case of constructs with randomly oriented collagen fibers, $C$ was chosen 0, i.e., no preferred fiber direction was considered.

\subsection{Simulations}

Simulations required a two-step approach. In the initial equilibration step, the implant and the cartilage were allowed to swell independently until equilibrium. During this swelling step, the implant was allowed to freely expand in radial and axial directions while the symmetry axis was confined in radial direction. The bottom plane of the native tissue region was confined in all directions to mimic the attachment of the cartilage to the subchondral bone. At the end of this step, we adjusted the pre-swollen implant size such that the swollen size of the implant exactly matched the gap region in the host cartilage, i.e., $L=6 \mathrm{~mm}$ in radius and $H=2.36 \mathrm{~mm}$ in height.

In the subsequent loading step, full integration between native tissue and the implant was prescribed using tie contact between them. Loading by the femoral condyle was simulated by using a rigid impermeable indenter with a curvature similar to the femoral condyle (Wilson et al. 2003). The contact between the femur and the tibia cartilage was assumed to be frictionless. Fluid outflow was only allowed at the parts of the articular surface that were not in contact with the femur. 
(a)

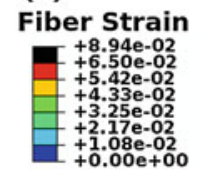

(c)

Volumetric Strain

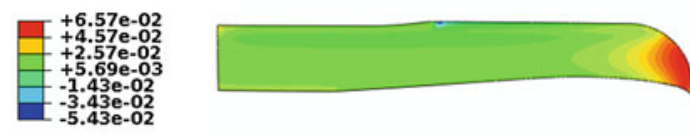

(b)

Deviatoric Strain

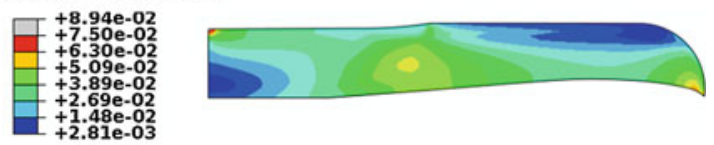

(d)

Contact Pressure (MPa)

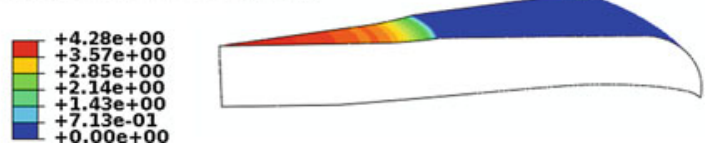

Fig. 2 a Peak fiber strain, $\mathbf{b}$ deviatoric strain, $\mathbf{c}$ volumetric strain and $\mathbf{d}$ contact pressure in native cartilage without implant

The bottom plane was confined in all directions, and the symmetry axis was confined in the radial direction. A ramp stance load of $162.5 \mathrm{~N}$ was applied in $1 \mathrm{~s}$, and this load was kept constant for $30 \mathrm{~s}$ to achieve a natural loading condition. This was followed by a ramp gait load of $568.75 \mathrm{~N}$ that was applied in $1 \mathrm{~s}$ to simulate the loads during a walking step (Wilson et al. 2006a; Morlock et al. 2001).

Swelling equilibrium and post-implantation condition of implants with different synthesis rates for PG's and collagen were simulated as follows. The equilibrium condition that was reached for the implant after the initial swelling step was dependent on the balance between swelling pressure and collagen stress. Consequently, a different equilibrium state was reached when it was assumed that all collagen was produced early, after which the implant started to swell, or whether swelling due to proteoglycan synthesis occurred first and collagen was added later, assuming late synthesis. We considered these effects by comparing three cases in which all, half, or one-third of the fibers were active in resisting swelling pressure in initial swelling equilibrium step (i.e., these fibers were synthesized early) and the remainder was not active in resisting swelling pressure in initial swelling equilibrium step (i.e., these fibers were synthesized late). In the model, zero strain was prescribed for that portion of the fibers that were inactive in resisting swelling pressure. Implants with 3/4 native collagen content and native ground substance stiffness were compared while the collagen orientation in the implant was varied.

\section{Results}

\subsection{Native tissue without implant}

In the loaded area of native tissue without implant, superficial collagen was strained on average $4 \%$ (Fig. 2a). Matrix in the middle and the deep zone was under compression, and therefore, collagen fibers remained unstrained in the loaded area, but they were strained by osmotic pressure in the unloaded periphery. Average matrix deviatoric strain was $2 \%$, and the strain was inhomogeneously distributed. Average matrix volumetric strain was $2.5 \%$ with rather homogeneous distribution under the femoral indenter (Fig. 2b, c). The contact pressure at the cartilage surface was 4.2 MPa (Fig. 2d), which is in range of the reported data in the literature (Mononen et al. 2011; Agneskirchner et al. 2004; Meyer et al. 2008; Marzo and Gurske-DePerio 2009; Morimoto et al. 2009).

\subsection{Implants with randomly oriented collagen fibers}

When TE cartilage with randomly oriented fibers, $1 / 4$ native collagen content and 1/2 the native ground substance stiffness were implanted in the central region of the cartilage, fibers in the deep zone experienced 12\% strain (Fig 3a top). Local deviatoric strain increased by $50 \%$ (Fig. 3 b top) and the average volumetric strain increased up to sevenfold (Fig. 3c top). Increasing the ground substance stiffness to that of the native tissue had negligible effect on the magnitude and distribution of the strains (Fig 3a-c middle), but it increased the interface shear stress in the middle zone (Fig. 3d middle). Keeping the ground substance stiffness to be $1 / 2$ that of native tissue but increasing the collagen content from $1 / 4$ to $3 / 4$ that of the native content reduced fiber strains in the deep zone (Fig. 3a bottom), deviatoric strain (Fig. 3b bottom), and volumetric strain (Fig. 3c bottom). Furthermore, the interface shear stress was considerably decreased (Fig. 3d bottom).

\subsection{Implants with horizontally oriented collagen fibers}

In the implant with horizontally oriented primary fibers and randomly oriented secondary fibers, $1 / 4$ native collagen content and $1 / 2$ of the ground substance stiffness of the native tissue, primary fibers experienced an inhomogeneous strain with peak tensile strains of $9 \%$ concentrated at the superficial and middle zone, ameliorating to $4 \%$ in the deep zone (Fig. $4 \mathrm{a}$ top). Compared to the implant with randomly oriented fibers, the distribution of the deviatoric strain was different with slightly higher maximum magnitude (Fig. 4b top), the distribution and magnitude of the volumetric strain were slightly 
Fig. 3 a Peak fiber strain, b deviatoric strain, c volumetric strain and $\mathbf{d}$ interface shear stress in implants with randomly oriented primary fibers consisting of $1 / 2$ native ground substance stiffness and $1 / 4$ native collagen content (top), native ground substance stiffness and $1 / 4$ native collagen content (middle), and $1 / 2$ native ground substance stiffness and $3 / 4$ native collagen content (bottom)

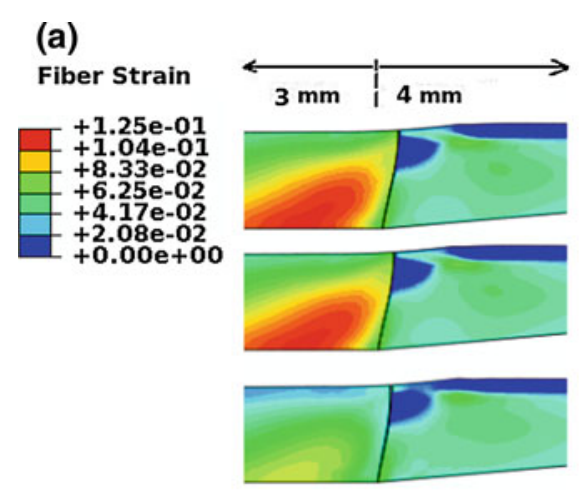

(c)

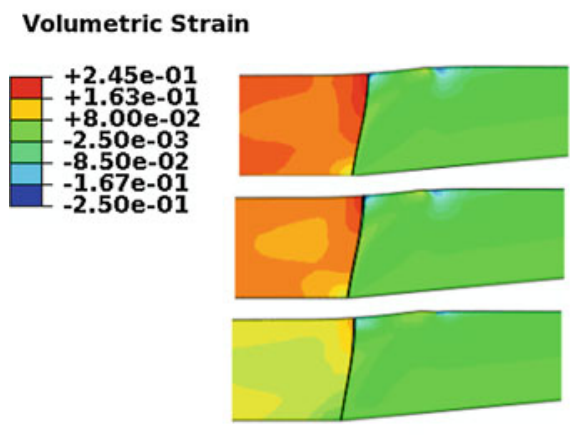

(b)

Deviatoric Strain

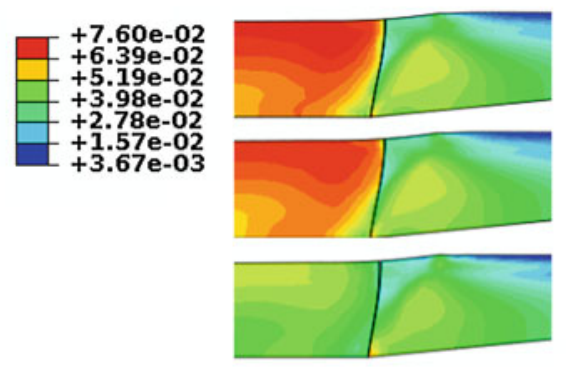

(d)

Shear Stress at Interface (MPa)

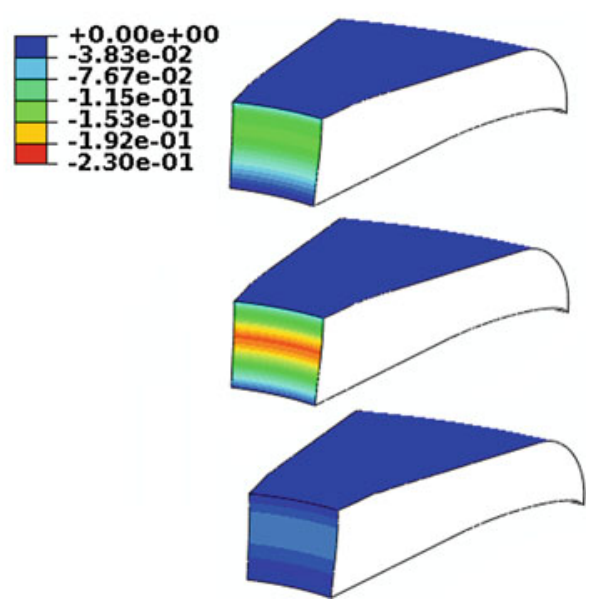

different (Fig. 4c top), and the interface shear stress had similar distribution with smaller magnitude in the middle and superficial zones. Increasing the ground substance stiffness to that of the native tissue had negligible effect on the magnitude and distribution of the strains (Fig. $4 \mathrm{a}-\mathrm{c}$ middle), but it increased the interface shear stress (Fig. $4 \mathrm{~d}$ middle). Increasing the collagen content from $1 / 4$ to $3 / 4$ native content while the ground substance stiffness was $1 / 2$ that of native tissue resulted in strain reduction, yet, with different distributions and magnitudes compared to the native tissue (Fig. 4 bottom).

\subsection{Implants with physiological collagen fibers}

In the implant with physiological predominant fibers and randomly oriented secondary fibers, $1 / 4$ native collagen content and $1 / 2$ of the ground substance stiffness of the native tissue, the distribution of primary fiber strain was similar to that of native tissue without implant. However, the magnitude of the fiber strain was higher (13\%) (Fig. 5a top). Increasing the ground substance stiffness to that of the native tissue had negligible effect on the strain magnitudes (Fig. 5a-c middle). Increasing the collagen content from $1 / 4$ to $3 / 4$ native content while the ground substance stiffness was $1 / 2$ that of native tissue decreased the fiber strains to $9 \%$ (Fig. 5a bottom).
The average volumetric strain was considerably decreased (Fig. 4c bottom).

\subsection{Implants with different synthesis rates of PGs and collagen}

Pre-implantation (Fig. 6a left) fiber strain in the implant with randomly oriented fibers, 3/4 native collagen content and native ground substance stiffness, increased from $4 \%$ when all collagen fibers were synthesized early (Fig. 6a top) to 5 and 6\% when half (Fig. 6a middle) and one-third (Fig. 6a bottom) of the fibers were synthesized early. This resulted in excessive collagen strains of 10 and $13 \%$ for the two later cases under loading, respectively (Fig. 6a right).

In the implant with horizontally oriented primary fibers and randomly oriented secondary fibers, $3 / 4$ native collagen content and native ground substance stiffness, pre-implantation (Fig. 6b left) peak fiber strain was concentrated toward the center of the implant increasing from $3 \%$ when all collagen fibers were synthesized early (Fig. 6b top) to 4 and $4.7 \%$ when half (Fig. 6b middle) and one-third (Fig. 6b bottom) of the fibers were synthesized early. This resulted in collagen strains of 7 and 9\% for the two later cases under loading (Fig. 6b right). 
(a)

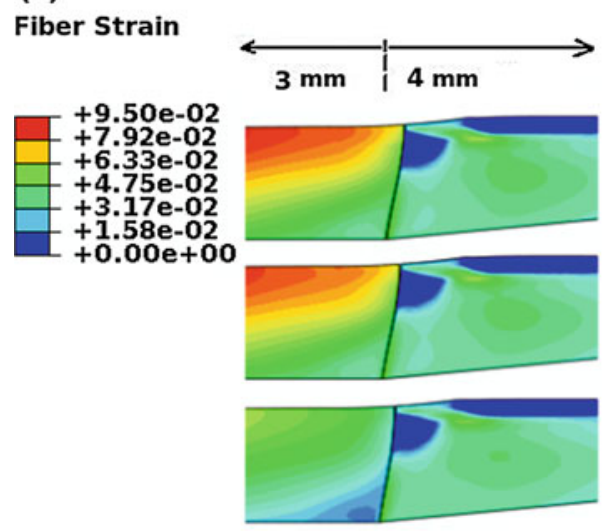

(c)

Volumetric Strain
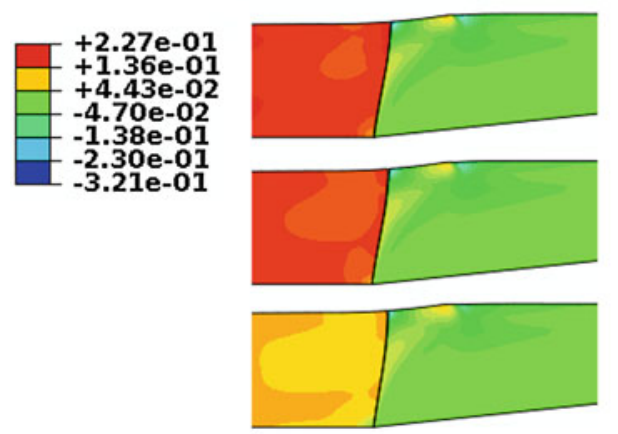

(b)

\section{Deviatoric Strain}

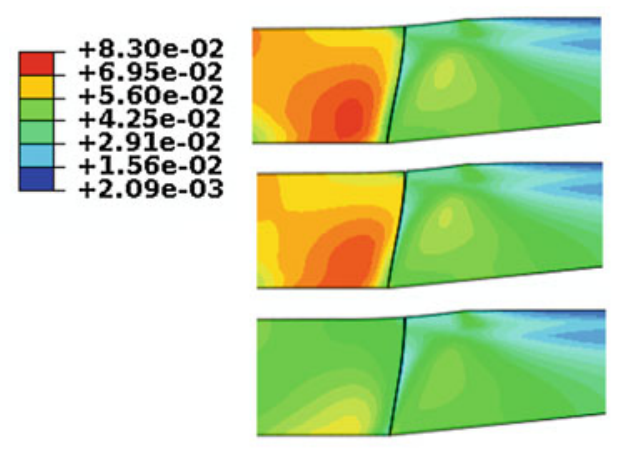

(d)

\section{Shear Stress at Interface (MPa)}

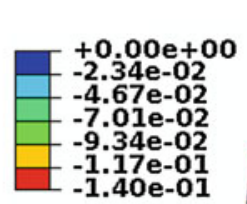

Fig. 4 a Peak fiber strain, b deviatoric strain, c volumetric strain and d interface shear stress in implants with horizontally oriented primary fibers consisting of $1 / 2$ native ground substance stiffness and $1 / 4$ native

In the implant with native-like primary fibers and randomly oriented secondary fibers, $3 / 4$ native collagen content and native ground substance stiffness, pre-implantation (Fig. 6c left) peak fiber strain was concentrated at the superficial layer increasing from $5 \%$ when all collagen fibers were synthesized early (Fig. 6c top) to 6 and $8 \%$ when half (Fig. 6c middle) and one-third (Fig. 6c bottom) of the fibers were synthesized early. Thus, late collagen synthesis resulted in excessive collagen strains of 11 and $13 \%$ for the two later cases under loading (Fig. 6c right).

\section{Discussion}

In the present study, the mechanical conditions inside and around a TE construct after implantation in intact cartilage collagen content (top), native ground substance stiffness and $1 / 4$ native collagen content (middle), and 1/2 native ground substance stiffness and $3 / 4$ native collagen content (bottom)

were analyzed to elucidate the relative importance of composition and structural organizations of the implant for its post-implantation mechanical performance. In particular, the importance of the implant ground substance stiffness, collagen content, and collagen architecture was investigated. This study showed that adverse parameters for each of these variables may lead to excessive mechanical loads, with collagen architecture being the most critical variable. Without physiological collagen architecture, a TE implant experiences a different distribution of the mechanical loads, independent of total tissue content. The most effective way to develop a tissue in which strains approach those in native tissue throughout the depth of the cartilage is to create a tissue with a physiological collagen structure. However, if physiological collagen architecture was formed but total collagen content was insufficient, then excessive strains were predicted to occur in the 
(a)
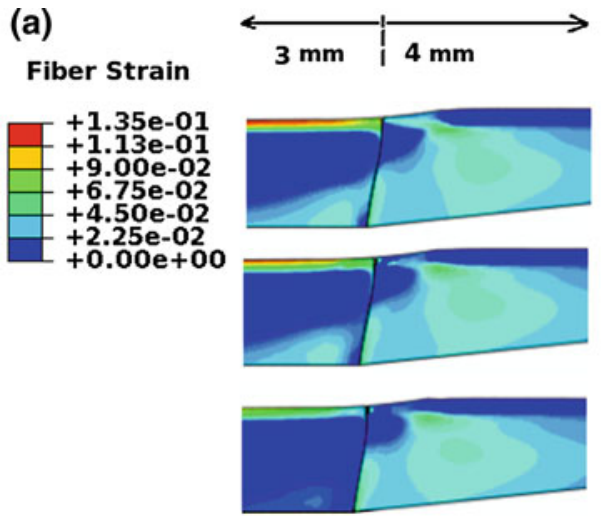

(c)
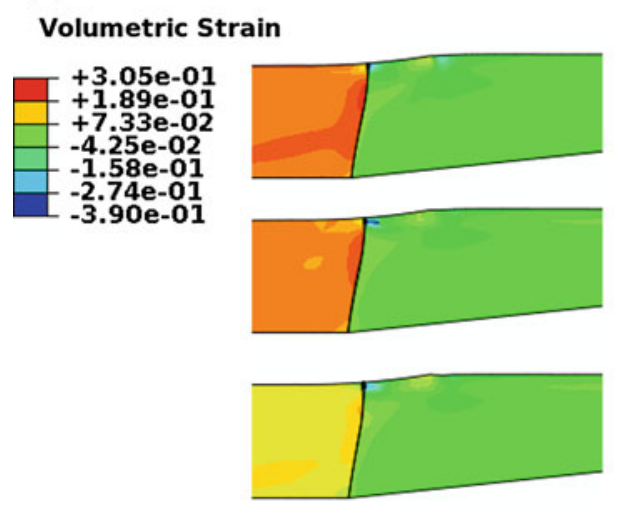

(b) Deviatoric Strain
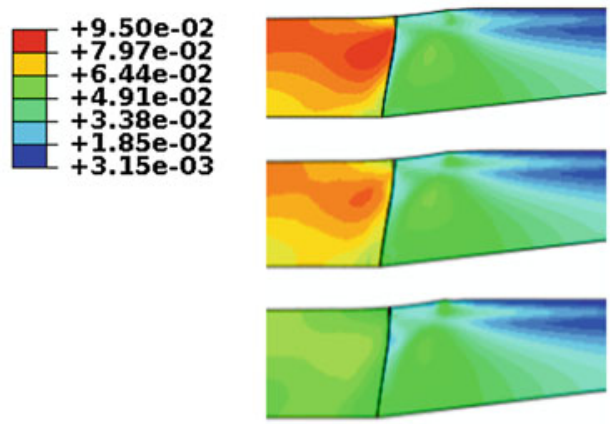

(d)

Shear Stress at Interface (MPa)

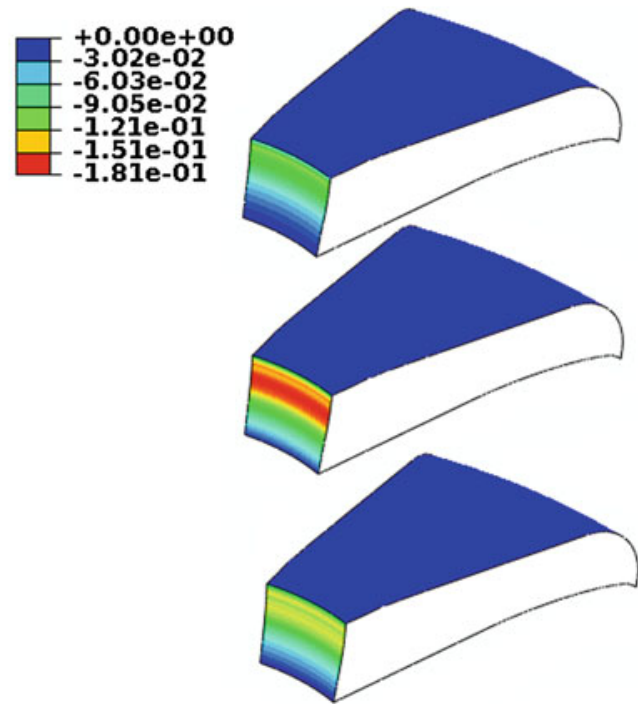

collagen content (top), native ground substance stiffness and 1/4 native collagen content (middle), and 1/2 native ground substance stiffness and $3 / 4$ native collagen content (bottom)

tissue. Both low and excessive straining may result in the degradation of the collagen network. It has been shown that collagen fibers undergo failure beyond 8-10\% strain (Wang 2006) and that cyclic compressive strains with rates in the range of $8-25 \% / \mathrm{s}$ cause immediate damage to collagen fibers (Thibault et al. 2002). When strain remains below 4\%, enzymatic degradation of collagen increases (Huang and Yannas 1977; Ruberti and Hallab 2005). Collagen network degeneration at the articular surface may lead to chondrocyte death, even at physiological loads/strains (Chen et al. 2003). In addition to such direct effects, changes in the collagen network may have indirect effects at the tissue and cell level. Because the PGs are retained by and swelling is restricted by the collagen network, loss of PGs and tissue swelling may occur with collagen failure resulting in tissue softening. Any of these changes to the collagen, PGs and water content of cartilage 
(a) Pre-implantation
swelling equilibruim

Post-implantation Under Loading Condition

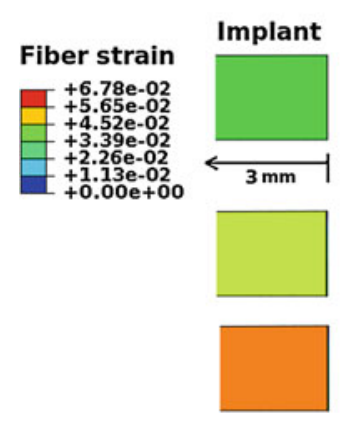

(c) Pre-implantation
swelling equilibruim

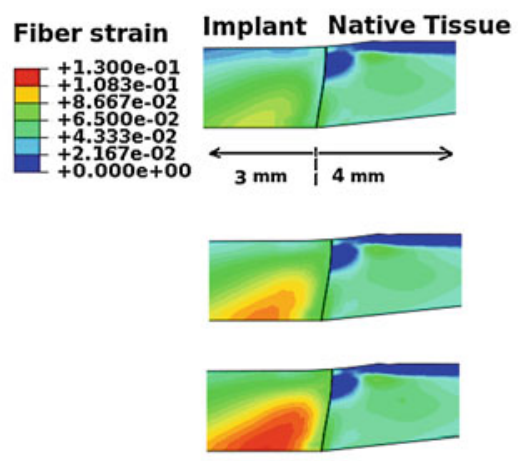

Post-implantation Under Loading Condition

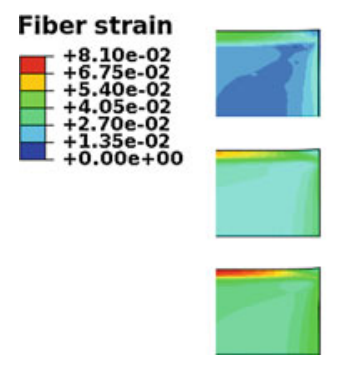

(b) Pre-implantation
swelling equilibruim
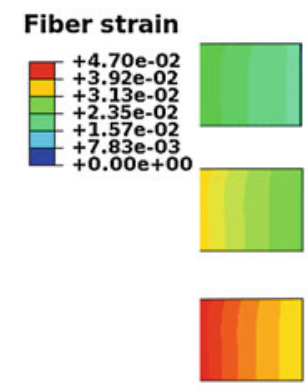

Post-implantation Under Loading Condition
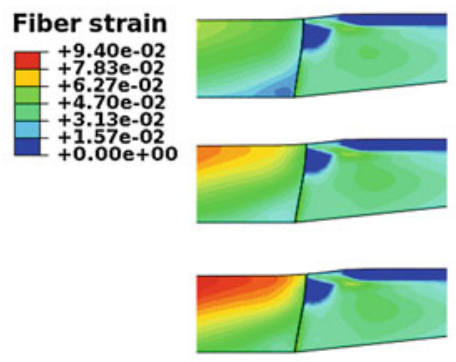

Fig. 6 Pre-implantation (left) and post-implantation (right) peak fiber strain in implants with randomly oriented fibers (a), horizontally oriented fibers (b), and physiological collagen network consisting of $1 / 2$ native ground substance stiffness and 3/4 native collagen content when all (top), half (middle), and one-third (bottom) of the collagen are synthesized early may alter the local mechanical environment of the cell, under the same physiological loads, thus altering cell anabolic/catabolic behavior and metabolism (Kurz et al. 2001; Smith et al. 2000).

Collagen content was found to be the most important parameter in determining interface shear stress. Assuming that high shear stresses at the interface between tissues will prohibit tissue integration, we postulate that implants with higher total collagen contents may have a higher chance to become integrated in the native host cartilage.

A second purpose of the present study was to evaluate how implant performance depends on the relationship between PG and collagen synthesis rates during TE. Interestingly, our pre-implantation free swelling results showed that rapid synthesis of PG's compare to that of collagen during cartilage TE leads to high strains in early synthesized collagen fibers while those fibers which are produced in later stages remain unstrained. This may explain experimentally different mechanical properties of TE construct with similar total PG and collagen contents (Bian et al. 2009). We predict that those fibers which are experiencing high strains at the moment of implantation are at danger of disruption when the tissue is mechanically loaded post-implantation, while those fibers which remain unstrained or experience a very low strain do not contribute much to the mechanical performance of the tissue. In addition, we may speculate that unstrained fibers may be degraded after implantation, because of their enhanced susceptibility to enzymatic degradation (Huang and Yannas 1977; Nabeshima et al. 1996; Ruberti and Hallab 2005). Such effects have not been reported before, and it may be worthwhile to explore this experimentally, or using models that include effects of transient development of cartilage matrix (Klisch et al. 2003; van Donkelaar and Wilson 2011). Hence, we postulate that a mismatch between collagen and PG synthesis rates in cartilage TE may lead to adverse mechanical conditions in the collagen fiber network. Modulation of osmotic pressure in constructs can be a solution to prevent excessive tension on newly synthesized collagen fibers. This may support the benefit of cartilage TE approaches in which glycosaminoglycan production is suppressed early during the TE process (Bian et al. 2009), to allow for first significant increase of collagen content.

In the present study, we focused on collagen fibers/cells susceptibility to disruption/damage due to high strains, which 
may occur upon join loading after implantation. At the time scale of months or years, biological adaptation involving tissue composition and realignment of collagen fibers may dominate the long-term clinical success rate of tissue engineering implants. However, for this to occur, the TE implants must be strong enough to survive immediate postimplantation loading.

In our simulations, $\mathrm{PG}$ content in the implant was assumed to be distributed homogeneously and to reach $75 \%$ of the native content, which is in the range of frequently reported amounts in TE studies (Mauck et al. 2003; Kelly et al. 2006). Future studies may consider the effects of the distribution of PG's for the mechanical properties of the constructs. In the present study, ground substance stiffness of the TE implants was varied independent from PG content to reflect the effects of using scaffolds with different mechanical stiffness.

Full integration has been reported in long-term experiments (Petersen et al. 2008), whereas others reported a cleft between the recipient articular cartilage and osteochondral plugs in goats and human (Lane et al. 2004; Horas et al. 2003). Our study suggests that these results may reflect a difference in the total content of collagen in the implants, which is important for the interface shear strains. In the present simulations, full implant-native tissue integration was assumed. Future numerical studies may address the effect of imperfect integration on the mechanical environment inside and around the engineered implants.

Implant size is a vital factor for clinical outcome of cartilage transplantation. The bigger the implant's dimensions, the larger the contact area between implant and surrounding subchondral bone. Because of more friction, higher forces are needed to displace an implant; thus, the implant is more stable (Kock et al. 2006; Duchow et al. 2000). Small diameter implants are less susceptible to damaging strains; yet, they are less stable. This may increase the chance of implant failure (Kock et al. 2006; Hurtig et al. 1999). Indeed, in clinical practice, treatment depends on defect size. Small defects are normally treated by microfracturing or abrasion, rather than transplantation. Accordingly, the implant diameter that we have considered in our study is chosen to be clinically relevant (Kock et al. 2006).

To answer our research questions, we required the use of a cartilage material model that includes reinforcement by collagen fibers in a depth-dependent organization, as well as a swelling proteoglycan network (Wilson et al. 2006b, 2007). For reasons of computational costs, we used a general axisymmetric model to represent the medial tibia plateau, similar to previous studies (Wilson et al. 2003, 2006a), rather than a more realistic three-dimensional geometry of the knee. Because we only compare simulations with the same loading and boundary conditions, we assume that this geometrical limitation does not affect our general conclusions.

\section{Conclusion}

Based on our results, we conclude that reproduction of the physiological collagen architecture enables sufficient loadbearing capacities of TE implants. However, such a structure is only functional if sufficient collagen is present. Therefore, we suggest that tissue engineering studies should best focus on collagen synthesis, rather than on engineering implants with abundant proteoglycan and enhanced ground substance stiffness.

In addition, we conclude that the faster synthesis rate of proteoglycans compared to that of collagen during TE may result in excessive swelling of the implant pre-implantation and may enhance the range of strains in the collagen network. We showed why both effects are undesirable to the mechanical functioning of a TE implant.

We believe that these aspects are worth to be considered in future tissue engineering studies, as they may result in mechanically superior engineered cartilage with enhanced long-term survival and improved integration.

Acknowledgments This study was supported with funding from the Dutch Technology Foundation STW (VIDI-07970).

Open Access This article is distributed under the terms of the Creative Commons Attribution License which permits any use, distribution, and reproduction in any medium, provided the original author(s) and the source are credited.

\section{References}

Agneskirchner JD, Hurschler C, Stukenborg-Colsman C et al (2004) Effect of high tibial flexion osteotomy on cartilage pressure and joint kinematics: a biomechanical study in human cadaveric knees. Arch Orthop Trauma Surg 124(9):575-584

Asanbaeva A, Masuda K, Thonar EJ-MA, Sah RL (2007) Mechanisms of cartilage growth modulation of balance between proteoglycan and collagen in vitro using chondroitinase ABC. Arthrit Rheum 56(1):188-198

Bian L, Crivello KM, Ng KW et al (2009) Influence of temporary chondroitinase $\mathrm{ABC}$-induced glycosaminoglycan suppression on maturation of tissue-engineered cartilage. Tissue Eng Part A 15(8):2065-2072

Buschmann MD, Gluzband YA, Grodzinsky AJ et al (1992) Chondrocytes in agarose culture synthesize a mechanically functional extracellular matrix. J Orthop Res 10(6):745-758

Chen C-T, Bhargava M, Lin PM, Torzilli PA (2003) Time, stress, and location dependent chondrocyte death and collagen damage in cyclically loaded articular cartilage. J Orthop Res 21:888-898

Duchow J, Hess T, Kohn D (2000) Primary stability of press-fitimplanted osteochondral grafts: influence of graft size, repeated insertion, and harvesting technique. Am J Sports Med 28:24-27

Eyrich D, Brandl F, Appel B et al (2007) Long-term stable fibrin gels for cartilage engineering. Biomaterials 28:55-65

Glaser C, Putz R (2002) Functional anatomy of articular cartilage under compressive loading Quantitative aspects of global, local and zonal reactions of the collagenous network with respect to the surface integrity. Osteoarthr Cartil 10(2):83-99 
Horas U, Pelinkovic D, Herr G (2003) Autologous chondrocyte implantation and osteochondral cylinder transplantation in cartilage repair. J Bone Joint Surg Am 85(2):185-192

Hu JC, Athanasiou KA (2006) A self-assembling process in articular cartilage tissue engineering. Tissue Eng 12:969-979

Huang C, Yannas IV (1977) Mechanochemical studies of enzymatic degradation of unsoluble collagen fibers. J Biomed Mater Res 11:137-154

Hurtig M, Evans P, Pearce S et al. (1999) The effect of graft size and number on the outcome of mosaic arthroplasty resurfacing: an experimental model in sheep. Transactions, 18th Annual Meeting of the Arthroscopy Association of North America, Vancouver, vol 18, pp 16-17

Kelly TAN, Ng KW, Wang CC et al (2006) Spatial and temporal development of chondrocyte-seeded agarose constructs in free-swelling and dynamically loaded cultures. J Biomech 39: 1489-1497

Khoshgoftar M, van Donkelaar CC, Ito K (2011) Mechanical stimulation to stimulate formation of physical collagen architecture in tissue-engineered cartilage; a numerical study. Comp Meth Biomech Biomed Eng 14(2):135-144

Kim TK, Sharma B, Williams CG et al (2003) Experimental model for cartilage tissue engineering to regenerate the zonal organization of articular cartilage. Osteoarthr Cartil 11:653-664

Klein TJ, Chaudhry M, Bae WC, Sah RL (2007) Depth-dependent biomechanical and biochemical properties of fetal, newborn, and tissue-engineered articular cartilage. J Biomech 40:182-190

Klisch SM, Chen SS, Sah RL (2003) A growth mixture theory for cartilage with application to growth-related experiments on cartilage explants. J Biomech Eng 125:169-179

Kock LM, van Donkelaar CC, Ito K (2010) Depth-dependent stimulation of chondrocytes in agarose by sliding indentation. In 56th annual meeting of the Orthopaedic Research Society, New Orleans, vol 35, p 810

Kock NB, van Susante JLC, Buma P et al (2006) Press-fit stability of an osteochondral autograft. Influence of different plug length and perfect depth alignment. Acta Orthopaed 77(3):422-428

Korhonen RK, Julkunen P, Wilson W, Herzog W (2008) Importance of collagen orientation and depth-dependent fixed charge densities of cartilage on mechanical behavior of chondrocytes. J Biomech Eng 130(2):021003

Korhonen RK, Wong M, Arokoski J et al (2002) Importance of the superficial tissue layer for the indentation stiffness of articular cartilage. Med Eng Phys 24:99-108

Kuo CK, Li WJ, Mauck RL, Tuan RS (2006) Cartilage tissue engineering: its potential and uses. Curr Opin Rheum 18:64-73

Kurz B, Jin M, Patwari P, Cheng D, Lark M, Grodzinsky A (2001) Biosynthetic response and mechanical properties of articular cartilage after injurious compression. J Orthop Res 19:1140-1146

Lane JG, Massie JB, Ball ST (2004) Follow-up of osteochondral plug transfers in a goat model: a 6-month study. Am J Sports Med 32(6): 1440-1450

Malda J, Woodfield T, van der Vloodt F et al (2005) The effect of PEGT/PBT scaffold architecture on the composition of tissue engineered cartilage. Biomaterials 26(1):63-72

Marzo JM, Gurske-DePerio J (2009) Effects of medial meniscus posterior horn avulsion and repair on tibiofemoral contact area and peak contact pressure with clinical implications. Am J Sports Med 37(1):124-129

Mauck RL, Wang CC-B, Oswald ES et al (2003) The role of cell seeding density and nutrient supply for articular cartilage tissue engineering with deformational loading. Osteoarthr Cartil 11: 879-890

Meyer EG, Baumer TG, Slade JM et al (2008) Tibiofemoral contact pressures and osteochondral microtrauma during anterior cruciate ligament rupture due to excessive compressive loading and internal torque of the human knee. Am J Sports Med 36(10): 1966-1977

Miot S, Scandiuccide Freitas P, Wirz D et al (2006) Cartilage tissue engineering by expanded goat articular chondrocytes. J Orthop Res 24:1078-1085

Mononen ME, Julkunen P, Toyras J et al (2011) Alterations in structure and properties of collagen network of osteoarthritic and repaired cartilage modify knee joint stresses. Biomech Model Mechanobiol 10(3):357-369

Morimoto Y, Ferretti M, Ekdahl M et al (2009) Tibiofemoral joint contact area and pressure after single- and double-bundle anterior cruciate ligament reconstruction. Arthroscopy 25(1):62-69

Morlock M, Schneider E, Bluhm A et al (2001) Duration and frequency of every day activities in total hip patients. J Biomech 34:873-881

Moutos FT, Freed LE, Guilak F (2007) A biomimetic three dimensional woven composite scaffold for functional tissue engineering of cartilage. Nat Mater 6(2):162-167

Nabeshima Y, Grood ES, Sakurai A, Herman JH (1996) Uniaxial tension inhibits tendon collagen degradation by collagenase in vitro. J Orthop Res 14:123-130

Nesic D, Whiteside R, Brittberg M et al (2006) Cartilage tissue engineering for degenerative joint disease. Adv Drug Deliv Rev 58:300-322

Ng KW, Ateshian GA, Hung CT (2009) Zonal chondrocytes seeded in a layered agarose hydrogel create engineered cartilage with depthdependent cellular and mechanical inhomogeneity. Tissue Eng A $15: 1-10$

Ng KW, Wang CC, Mauck RL et al (2005) A layered agarose approach to fabricate depth-dependent inhomogeneity in chondrocyte-seeded constructs. J Orthop Res 23:134-141

Noth U, Steinert AF, Tuan RS (2008) Technology insight: adult mesenchymal stem cells for osteoarthritis therapy. Nat Clin Pract Rheum 4:371-380

Owen JR, Wayne JS (2006) Influence of a superficial tangential zone over repairing cartilage defects: implications for tissue engineering. Biomech Model Mechanobiol 5:102-110

Petersen JP, Ueblacker P, Goepfert C et al (2008) Long term results after implantation of tissue engineered cartilage for the treatment of osteochondral lesions in a minipig model. J Mater Sci Mater Med 19:2029-2038

Risbud MV, Sittinger M (2002) Tissue engineering: advances in in vitro cartilage generation. Trend Biotech 20:351-356

Ruberti JW, Hallab NJ (2005) Strain-controlled enzymatic cleavage of collagen in loaded matrix. Biochem Biophys Res Commun 336:483-489

Schek RM, Taboas JM, Segvich SJ et al (2004) Engineered osteochondral grafts using biphasic composite solid free-form fabricated scaffolds. Tissue Eng 10:1376-1385

Scotti C, Buragas MS, Mangiavini L et al (2007) A tissue engineered osteochondral plug: an in vitro morphological evaluation. Knee Surg Sports Traumatol Arthrosc 15:1363-1369

Shirazi R, Shirazi-Adl A (2008) Deep vertical collagen fibrils play a significant role in mechanics of articular cartilage. J Orthop Res 26:608-615

Shirazi R, Shirazi-Adl A, Hurtig M (2008) Role of cartilage collagen fibrils networks in knee joint biomechanics under compression. $\mathrm{J}$ Biomech 41:3340-3348

Smith RL, Trindade MCD, Ikenoue T et al (2000) Effects of shear stress on articular chondrocyte metabolism. Biorheology 37:95-107

Thibault M, Poole AR, Buschmann MD (2002) Cyclic compression of cartilage/bone explants in vitro leads to physical weakening, mechanical breakdown of collagen and release of matrix fragments. J Orthop Res 20:1265-1273

Torzilli P (1993) Effects of temperature, concentration and articular surface removal on transient solute diffusion in articular cartilage. Med Biol Eng Comp 31(Suppl):S93-S98 
Torzilli P, Dethmers D, Rose D, Schryuer H (1983) Movement of interstitial water through loaded articular cartilage. J Biomech 16(3):169-179

van Donkelaar CC, Wilson W (2011) Mechanics of chondrocyte hypertrophy. Biomech Model Mechanobiol. doi:10.1007/s10237-0110340-0

Wang JHC (2006) Mechanobiology of tendon. J Biomech 39: $1563-1582$

Wilson W, Driessen NJB, van Donkelaar CC et al (2006a) Prediction of collagen orientation in articular cartilage by a collagen remodeling algorithm. Osteoarthr Cartil 14:1196-1202

Wilson W, Huyghe JM, van Donkelaar CC (2006b) A compositionbased cartilage model for the assessment of compositional changes during cartilage damage and adaptation. Osteoarthr Cartil 14: $554-560$
Wilson W, Huyghe JM, van Donkelaar CC (2007) Depth-dependent compressive equilibrium properties of articular cartilage explained by its composition. Biomech Model Mechanobiol 6:43-53

Wilson W, van Donkelaar CC, van Rietbergen B et al (2004) Stresses in the local collagen network of articular cartilage: a poroviscoelastic fibril-reinforced finite element study. J Biomech 37:357-366

Wilson W, van Rietbergen B, van Donkelaar CC, Huiskes R (2003) Pathways of load-induced cartilage damage causing cartilage degeneration in the knee after meniscectomy. J Biomech 36: $845-851$ 